\title{
B Physics, BTeV, and all that
}

\author{
Joel N. Butler \\ Fermi National Accelerator Laboratory, Batavia, Illinois 60510-0500 USA \\ Presented at a panel discussion at Beauty 2002, in Santiago de \\ Compostela, Spain
}

\begin{abstract}
The status of "flavor physics" in our pursuit of knowledge in elementary particle physics is discussed. Then, the BTeV experiment, planned for the Fermilab Tevatron collider, is described briefly and its physics reach is discussed. Comparisons are made to the current $B$ physics experiments at $e^{+} e^{-}$facilities and to the $\mathrm{LHCb}$ experiment, planned for the Large Hadron Collider at CERN.
\end{abstract}




\section{Introduction}

This talk has two related themes. The first is a general discussion of the "stature" of the study of $B$ physics, and heavy flavors in general, in the world of elementary particle physics. The second is a brief presentation of the goals and status of the BTeV [1] experiment, which was proposed for the Fermilab Tevatron and approved by Fermilab more than two years ago but which is still awaiting a decision to proceed by the US Department of Energy (DOE).

\section{B Physics and Quark Flavor Physics - General Considerations}

Recently, there has been dramatic and exciting progress in the area of experimental studies of $\mathrm{CP}$ violation in the decays of heavy quarks:

- KTeV [2] and NA48 [3] have made major advances in reducing the statistical and systematic uncertainties in $\epsilon^{\prime} / \epsilon$ and have finally established the existence of "direct $\mathrm{CP}$ violation" in kaon decays.

- BaBar [4] and BELLE [5] have conclusively established CP violation in $B_{d}$ decays through the measurement of $\sin 2 \beta$, which is clearly shown to be many standard deviations away from zero. They will continue to study other $\mathrm{CP}$ violating phenomena and rare decays of $B_{d}$ and $B_{u}$ for many years, eventually limited by the number of $B$ 's that their machines can produce.

The Fermilab experiments CDF and D0 are expected to acquire new results on $B_{s}$ mixing and $\mathrm{CP}$ violation in $B_{d, u}$ and $B_{s}$ decays, in Tevatron Collider Run II [6].

After this phase of investigations, there will still be much work to do and that is where the $\mathrm{BTeV}$ experiment, discussed below and elsewhere in this workshop [7], is expected to excel.

In the Standard Model (SM) of Elementary Particle Physics, CP violation in quark decays arises from the quark mixing matrix, called the CKM matrix, [8]. The CKM matrix is often shown in a very useful form, the Wolfenstein parametrerization [9], which clearly exhibits the "generation" hierarchy of quark decays:

$$
\left(\begin{array}{ccc}
1-\frac{1}{2} \lambda^{2}\left(V_{u d}\right) & \lambda\left(V_{u s}\right) & A \lambda^{3}\left[\rho-i \eta\left(1-\frac{1}{2} \lambda^{2}\right)\right]\left(V_{u b}\right) \\
-\lambda\left(V_{c d}\right) & 1-\frac{1}{2} \lambda^{2}-i \eta A^{2} \lambda^{4}\left(V_{c s}\right) & A \lambda^{2}\left(1+i \eta \lambda^{2}\right)\left(V_{c b}\right) \\
A \lambda^{3}[1-\rho-i \eta]\left(V_{t d}\right) & -A \lambda^{2}\left(V_{t s}\right) & 1\left(V_{t b}\right)
\end{array}\right)
$$

The key point is that in the SM, ALL manifestations of CP violation in quark decays depend on the parameter $\eta$, which must be non-zero for them to exist at all. A second point is that quarks prefer to decay into other quarks within their own generation and the decay rate is reduced when there is a "generation gap". This is expressed by the the parameter $\lambda \approx 0.22$, the sine of the Cabbibo angle, that appears in the matrix elements connecting intergenerational decays. 
The smallest number of generations for which a weak phase is permitted by the required unitarity of the mixing matrix is three. In the Wolfenstein parameterization, to order $\lambda^{3}$, the CP violating parameter $\eta$ appears in the "small" matrix elements $V_{t d}$ and $V_{u b}$ between the first and third generation.

If the CKM matrix is the reason for all $\mathrm{CP}$ violation in quark decays, then all $\mathrm{CP}$ violating decays are related to each other because there is only one parameter which drives this phenomenon. (In fact, if we understood the issues associated with the binding of the quarks into hadrons, the observation of any one CP violating decay would allow us to predict all the others.)

We are compelled to ask the question whether the CKM mechanism is the complete explanation of the $\mathrm{CP}$ violation in quark decays or whether there are other sources, which could come from new physics beyond the SM appearing in loops or box diagrams of the decays.

One can best characterize the current situation as follows:

- The Standard Model CP violation is unique, predictive, and testable.

- Even now after all the recent progress, CP violation is one of the least tested aspects of the SM.

- Almost any extension of the SM has new sources of CP violation.

- The observed baryon asymmetry of the universe requires new sources of CP violation $[10]$

It is therefore an UNAVOIDABLE conclusion that the SM picture of CP violation is incomplete. $\mathrm{CP}$ violation is an excellent probe for new physics and one which may well be complementary to direct searches. The clear message is that we must challenge the SM predictions of CP violation on every front, constantly looking for discrepancies which could indicate new phenomena.

We may ask why this problem does not generate more enthusiasm in the particle physics community. In my view, this is due to our failure to connect more specifically to New Physics and the "big problems" in a field which is increasingly obsessed with one particular problem, electroweak symmetry breaking. In particular, there are two main missing ingredients:

- the lack of a comprehensive set of calculations which relate specific $B$ physics measurements to sensitivities to New Physics scenarios in a quantitative way; and

- generally, the lack of one of more "theories of flavor" which pinpoint decisive measurements. (For example, string theory has very little to say on the whole topic of flavors)

The HEP community is most enthusiastic and assigns highest priority and urgency to areas where theory and experiment are seen as converging to produce definitive tests that will lead to assured near-term progress. When a field is mainly experiment driven, it 
is assigned lower priority even though new, unanticipated experimental results may be exactly what are needed to stimulate the theory.

Table 1 lists some of the key quantities which need to be measured, or measured better, to confront the SM of CP violation. The angles $\alpha, \beta$, and $\gamma$ are the angles of the CKM triangle [11]. The angle $\chi$ is defined as

$$
\chi=\arg \left(\frac{V_{c s}^{*} V_{c b}}{V_{t s}^{*} V_{t b}}\right)
$$

\begin{tabular}{||l|l||}
\hline \hline Physics Quantity & Decay Mode \\
\hline $\sin 2 \alpha$ & $B^{o} \rightarrow \rho \pi \rightarrow \pi^{+} \pi^{-} \pi^{o}$ \\
$\cos 2 \alpha$ & $B^{o} \rightarrow \rho \pi \rightarrow \pi^{+} \pi^{-} \pi^{o}$ \\
$\operatorname{sign}(\sin 2 \alpha)$ & $B^{o} \rightarrow \rho \pi, B^{o} \rightarrow \pi^{+} \pi^{-}$ \\
$\sin \gamma$ & $B_{s} \rightarrow D_{s} K^{-}$ \\
$\sin \gamma$ & $B^{+} \rightarrow D^{o} K^{+}$ \\
$\sin \gamma$ & $B \rightarrow K \pi$ \\
$\sin \gamma$ & $B \rightarrow \pi^{+} \pi^{-}, B_{s} \rightarrow K^{+} K^{-}$ \\
$\sin 2 \chi$ & $B_{s} \rightarrow J / \psi \eta^{\prime}, J / \psi \eta$ \\
$\sin 2 \beta$ & $B^{o} \rightarrow J / \psi K_{s}$ \\
$\sin 2 \beta$ & $B^{o} \rightarrow \phi K_{s}, \eta^{\prime} K_{s}, J / \psi \phi$ \\
$\cos 2 \beta$ & $B^{o} \rightarrow J / \psi K^{*}, B_{s} \rightarrow J / \psi \phi$ \\
$x_{s}$ & $B_{s} \rightarrow D_{s} \pi^{-}$ \\
$\Delta \Gamma$ for $B_{s}$ & $B_{s} \rightarrow J / \psi \eta^{\prime}, K^{+} K^{-}, D_{s} \pi^{-}$ \\
\hline \hline
\end{tabular}

Table 1: Key Physics Quantities and B Decay Modes

From the table, one sees that about half the important measurements involve $B_{s}$ decays and about half of the key decay modes have $\gamma^{\prime}$ s or $\pi^{o}$ 's in the final state. This provides strong requirements on the experiments that need to be mounted to address these issues.

There are two general approaches to attacking the SM through the study of CP violation in $B$ decays.

The first is to look for inconsistencies in the SM picture. For example, the SM model makes the following prediction:

$$
\sin \chi=\frac{\left|V_{u s}\right|^{2}}{\left|V_{u d}\right|^{2}} \times \frac{\sin \beta \sin \gamma}{\sin (\beta+\gamma)}
$$

The second is to relate specific decay rate deviations or limits of such deviations to specific models and use the measured rates to either establish the possibility of new contributions or rule them out (perhaps ruling out only some part of an available parameter space). Models which exhibit possible non-SM CP violation include: SUSY and multiHiggs models, left-right symmetric models, models with extra down-type singlet quarks, models with flavor changing neutral current decays of the $Z$ boson, and models containing 
extra dimensions, to name a few. Of course, definite predictions would need to be calculated to carry out this exercise. It is worth noting that $B$ mixing and decays involving loops already are used to provide constraints on the masses of possible charged Higgs bosons and, in the context of SUSY, parameters such as $\tan \beta_{S U S Y}$.

So, to summarize, we need

- for our theorists to sharpen the relation between specific measurements and New Physics Scenarios, i.e. for each model, what range of physics parameters can be probed given a reasonably achievable level of experiment sensitivity to specific $B$ decays, asymmetries, etc.; and

- a comprehensive theory of flavor, which would presumably relate the CKM mass matrix to quark masses and might shed light on the relation of the CKM matrix to our other "flavor mixing matrix" in the neutrino sector, the MNS matrix [12].

If at least the first of these steps is not achieved, I fear that the status and stature of $B$ experiments will continue to be lower than I believe they deserve.

\section{$2 \mathrm{BTeV}$}

$\mathrm{BTeV}[1]$ is an experiment to study $\mathrm{CP}$ violation and rare decays of particles containing b-quarks at the Fermilab Tevatron collider. It is has already been discussed at this conference. A schematic layout of the detector is given in Figure 1. This version is a "single arm spectrometer" which covers angles from $10 \mathrm{mr}$ to $300 \mathrm{mr}$ with respect to the antiproton beam. The previous version was symmetric about $z=0$ and covered the angular region from $10 \mathrm{mr}$ to $300 \mathrm{mr}$ with respect to BOTH the proton and antiproton beams. The scope was reduced to a single arm in order to save money to better match the cost of BTeV to Fermilab and DOE funding realities.

Key design features of $\mathrm{BTeV}$ are:

- a dipole centered on the Interaction Region (IR), which gives BTeV large angular coverage while keeping it very compact.

- a precision vertex detector based on planar arrays of silicon detectors, centered on the IR and, therefore, immersed in the field of the dipole.

- a detached vertex trigger implemented at Level 1 (the lowest level of the trigger), based on the pixel detector and taking advantage of the presence of the strong magnetic field at the IR.

- strong particle identification based on Ring Imaging Cerenkov counters. Many of the states that are of interest in this phase of $B$ physics will only be cleanly separable from other states if this capability exists.

- a highly segmented Lead Tungstate electromagnetic calorimeter, which provides outstanding photon and $\pi^{o}$ reconstruction. 
BTeV Detector Layout

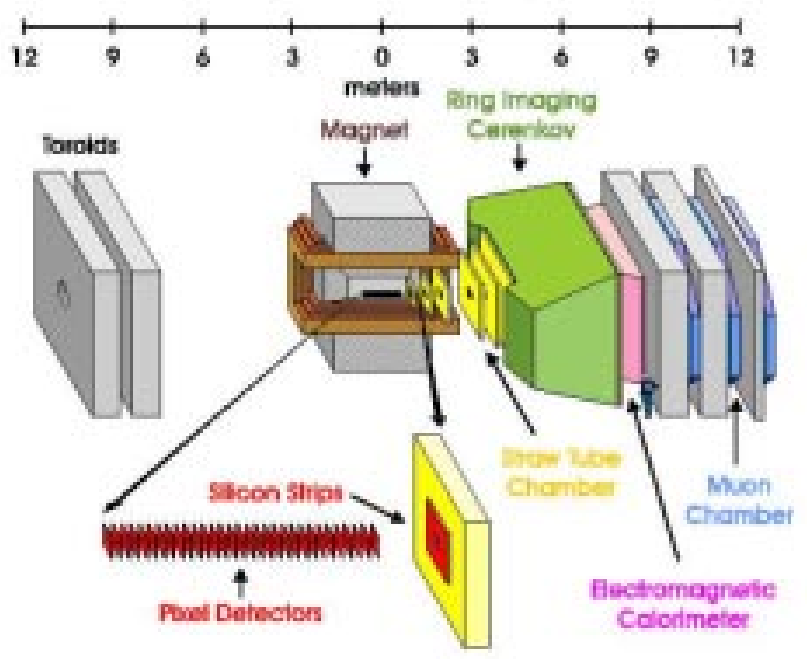

Figure 1: Schematic of the Single Arm Version of the BTeV Detector

- a high rate data acquisiton system which allows BTeV to record almost all $B$ decays, regardless of final state, based only on the topological requirement of evidence for a detached vertex. This means that $\mathrm{BTeV}$ will record decays that might not be recognized to be important but which may later turn out to be.

The current status of BTeV is that it is awaiting review by the Particle Physics Project Prioritization Panel, known as P5, which has been formed in November of 2002 to determine the priority of midlevel projects in the US HEP program.

\subsection{Comparison of BTeV to $e^{+} e^{-} B$ Factories}

The E2 Working Group [15] at Snowmass 2001 established that an $e^{+} e^{-}$asymmetric $B$ factory running on the $\Upsilon(4 \mathrm{~S})$ with a luminosity of $10^{35} / \mathrm{cm}^{2}$-s, the endpoint of upgrades to existing machines, would reconstruct only $1 / 10$ as many $B_{d}$ and $B_{u}$ decays as would $\mathrm{BTeV}$ per year of running. Of course, they would not learn anything about $B_{s}$ decays or the decay of other $B$ hadrons. The E2 group concluded that a machine with a luminosity of $10^{36} / \mathrm{cm}^{2}$-s would be required for $e^{+} e^{-}$to be competitive in the study of $B_{d}$ and $B_{u}$. This would require a new machine (although not a new tunnel) and signficant accelerator physics problems would have to be solved through extensive R\&D. A very substantial investment of accelerator physics resources would have to be made to carry out this $\mathrm{R} \& \mathrm{D}$, to design and construct the machine, and to commission it. Moreover, the BaBar detector would have to be essentially rebuilt to operate at the higher rate and to tolerate more severe backgrounds. The technologies required for this new detector, called "SuperBaBar" to achieve efficiencies and resolutions that are comparable to what BaBar has today do not yet exist and would need to be developed, if possible, in an extensive program of 
detector R\&D. The cost of the new machine and upgraded detector, if it can, in fact, be built, is expected to be very large, several times that of BTeV or $\mathrm{LHCb}$.

\subsection{Comparison of $\mathrm{BTeV}$ to $\mathrm{LHCb}$}

There have by now been several comparisons of BTeV and LHCb. Table 2 shows rates and signal-to-background ratios for the decays

$$
\begin{aligned}
& B_{o} \rightarrow \rho^{ \pm} \pi^{\mp} \rightarrow \pi+\pi^{-} \pi^{o} \\
& B_{o} \rightarrow \rho^{o} \pi^{o} \rightarrow \pi+\pi^{-} \pi^{o}
\end{aligned}
$$

This decay is considered one of the best ways to determine the CKM angle $\alpha$ via a detailed Dalitz plot analysis [16]. Because of its superior electromagnetic calorimetry, BTeV is a factor of 2.5 better in total signal events but has an actual advantage of a factor of 4 when BTeV's superior signal to background is taken into account.

It is also worth noting that BTeV's trigger accepts a much wider range of $B$ decays than LHCb's and BTeV's data acquisition system is capable of recording many more events than LHCb's. This means that BTeV is not tuned for a specific set of "theoretically desirable" decays but can also look for the unexpected. BTeV will also have a much larger sample of charm decays to study for $\mathrm{CP}$ violation, mixing, and other rare phenomena.

It is worth noting that $\mathrm{BTeV}$ and $\mathrm{LHCb}$ are complementary in several ways. $\mathrm{LHCb}$ benefits by a factor of 5 more cross section. BTeV makes up for this with its more aggressive technology - the pixel detector, the more sophisticated trigger, and the superior calorimeter. BTeV also has to cover a smaller momentum range, which makes it more compact.

KTeV and NA48 and BELLE and BaBar drove each other to build their detectors on time and to analyze their data in a timely fashion. Each benefitted from the efforts of the other. These two experiments, BTeV and $\mathrm{LHCb}$, can take the study of CP violation in $B$ decays to new heights and will reinforce as well as complement each other.

\begin{tabular}{||l|c|c|c|c|c||}
\hline \hline Mode & $\begin{array}{c}\text { Branching } \\
\text { Ratio }\end{array}$ & $\begin{array}{c}\text { BTeV } \\
\text { Yield }\end{array}$ & $\begin{array}{c}\text { BTeV } \\
\text { S/B }\end{array}$ & $\begin{array}{c}\text { LHCb } \\
\text { Yield }\end{array}$ & $\begin{array}{c}\text { LHCb } \\
\text { S/B }\end{array}$ \\
\hline$B^{o} \rightarrow \rho^{ \pm} \pi^{\mp}$ & $2.8 \times 10^{-5}$ & 5400 & 4.1 & 2140 & 0.8 \\
$B^{o} \rightarrow \rho^{o} \pi^{o}$ & $0.5 \times 10^{-5}$ & 776 & 0.3 & $\begin{array}{c}880 \text { "naive" } \\
\text { w.o. background }\end{array}$ & $\begin{array}{c}<0.05 \\
\text { my estimate }\end{array}$ \\
\hline \hline
\end{tabular}

Table 2: $B^{o} \rightarrow \rho \pi$ : Event Yields and Signal-to-Background ratios (S/B) for BTeV and $\mathrm{LHCb}$ for one year of data taking

\subsection{Issues in the US HEP Program}

In the US, while our HEP program is overburdened today, we have no new DOE funded domestic accelerator-based construction project after 2006. With $50 \%$ of the US HEP 
community already planning to work on CMS and ATLAS at the LHC, the US faces an unprecedented crisis when it loses the energy frontier around 2008, the start of the LHC physics program. Without clear domestic alternatives, experimental high energy physicists who do not wish to work at CERN on ATLAS or CMS, experiments whose lifetime is projected to be 20 years, will likely go into non-accelerator based fields. This suggests that the physicist strength to undertake a large scale accelerator project, such as a Linear Collider or a neutrino storage ring/muon collider, may not be present in the US program if it is needed several years down the road. BTeV, with its advanced technology and broad physics program, can supply crucial hardware experience and numerous data analysis topics for theses to train the next generation of young physicists.

In the US, accelerator physics resources are also in very short supply. While BTeV does cost money, it does not require much additional effort from accelerator physicists since it places lower luminosity requirements on the Tevatron than does RUN II. In addition to providing excellent physics opportunties, BTeV is an effective use of investments the nation has made (and expects to make in the next few years) in the Tevatron.

\subsection{Possible BTeV Schedule}

Given the problems associated with an experiment which must follow Tevatron RUN IIB, there are always concerns about the BTeV schedule. At present, the IR for BTeV at $\mathrm{C} 0$ is supposed to use quadrupoles reclaimed from either D0 or B0. Here is a "realistic" view of what could happen:

- BTeV could be built by 2008, with substantial portions in place by 2007.

- $\mathrm{BTeV}$ is designed so components can be installed on the fly a little at a time on collider down days.

- During RUN IIB, BTeV can have collisions at low luminosity, $10^{30}$, at the end of stores, simply by having the separators turned off.

- When low luminosity colliding beam operation is not possible, we can debug detectors on flux from a wire target in the beam halo.

- We can be commissioned before the final IR is complete. This is worth at least a year, if not more.

The character of this physics is that it unfolds gradually as statistics are accumulated over a few years. In the end small differences in the starting time can be overcome by a superior detector. Even if we did start late with respect to LHCb, we have a sufficient advantage in some KEY states that we could rapidly catch up, e.g. $4 \mathrm{x}$ better in $\rho-\pi$. We assume that the moment when the transition to $\mathrm{BTeV}$ will be made will be determined by physics considerations with due respect to the laws of statistics - i.e. that RUN IIB will end when the "doubling time" becomes too long. However, Fermilab will begin, based on the PAC recommendation, to think about a "plan B" involving the construction of new 
magnets for $\mathrm{C} 0$, in case the physics of RUN IIB dictates that the two existing detectors continue.

\section{Concluding Remarks with respect to Flavor Physics and $\mathrm{BTeV}$}

Referring back to the first theme of this talk, we hope and expect that theoretical developments will help to establish the study of $\mathrm{CP}$ violation and rare decays of states containing heavy quarks as an effective tool in detecting and learning about new physics beyond the SM. This will improve the stature of these investigations and hopefully increase their priority.

BTeV can make critical contributions to our knowledge of CP Violation as attention turns from initial observations to the work of finding out if the Standard Model explanation is correct and complete. BTeV is not just doing Standard Model physics. It is sensitive enough to reveal new phenomena and to learn something about them. BTeV makes excellent use of an existing domestic HEP facility in which there has and will have been a huge investment but doesn't overtax precious accelerator $R \& D$ resources that will be needed to develop projects for the somewhat more distant future. The detector R\&D projects are critical to developing the technologies that will make these experiments possible and provide excellent and much needed training for future generations of experimental physicists. The work will insure that BTeV will succeed and will increase the likelihood that it can be done on schedule and on budget. The large range of topics that can be explored with the BTeV detector will provide many exciting, high impact thesis topics. $\mathrm{BTeV}$ is a necessary part of US HEP's response to the looming "crisis of 2008". Hopefully, BTeV will form a key part of a world class accelerator-based domestic flavor physics program after the LHC takes firm possession of the energy frontier and will serve as a "bridge" to new large machine facility in the US in the more distant future.

\section{Acknowledgement}

This work was supported in part by Fermilab, which is operated by Universities Research Association Inc. under Contract No. DE-AC02-76CH03000 with the United States Department of Energy.

\section{References}

[1] see http://www-btev.fnal.gov/public/hep/general/proposal/index.shtml, "BTeV Proposal Update", March 2002.

[2] KTeV Collaboration, A. Alavi-Harati et al., Phys. Rev. Lett. 83, 22-27 (1999)

[3] NA48 Collaboration, A. Lai et al., Eur. Phys. J. C22, 231-254 (2001) 
[4] BaBar Collaboration, B. Aubert et al., Phys. Rev. Lett., 89, 201802 (2002)

[5] BELLE Collaboration, K. Abe et al., Phys. Rev. D66, 071102(R) (2002)

[6] B Physics at the Tevatron Run II and Beyond, Fermilab-PUB-01/197, December 2001

[7] The following talks about BTeV were presented at this conference: The BTeV Trigger, Charles Newsom; BTeV - Lepton, Hadron, and Photon Identification, Rob Kutschke; BTeV - Overview, Status, and Prospects, Rob Kutschke

[8] M. Kobayashi and T. Masakawa, Prog. Theor. Phys. 49, 652 (1973)

[9] L. Wolfenstein, Phys. Rev. Lett. 51, 1945 (1983)

[10] C. Jarlskog, Z. Physik C29, 491 (1985); Phys. Rev. Lett. 55, 1039 (1985); M.E. Shaposhnikov, Nucl. Phys. B287, 757 (1087); B299, 797 (1988)

[11] L.L. Chau and W.Y. Keung, Phys. Rev. Lett 53, 1802 (1984); M. Gronau and J. Schechter, Phys. Rev. Lett. 54, 385 (1985); M. Gronau, R. Johnson, and J. Schechter, Phys. Rev. D32 3062 (1985); C. Jarlskog, in Physics at L.E.A.R with Low Energy Antiprotions, Villars-surOllon, Switzerland, 1987, edited by C. Amsler et al. (Harwood, Chur,Switzerland, 1988); J.D. Bjorken and I. Dunietz, Phys. Rev. D36, 2109 (1987)

[12] S.P. Mikheyev and A. Smirnov, Yad. Fiz. 42, 1441 (1985) Sov. J.Nucl.Phys 42, 913 (1986), Nuovo Cim., C9, 17 (1986); S.P. Rosen and J. Gelb, Phys. Rev. D34, 969 (1986); S. Parke, Phys. Rev. Lett. 57, 1275 (1986); W. Haxton, Phys. Rev. Lett. 57, 1271 (1986); J. Pantaleone and T.K. Kuo, Rev. Mod. Phys. 61, 937 (1989)

[13] see http://www-btev.fnal.gov/public/hep/general/proposal/index.shtml, "Preliminary TDR", May 1999

[14] see http://www-btev.fnal.gov/public/hep/general/proposal/index.shtml, "BTeV Proposal", May 2000

[15] Z. Zhao, et al., Report of Snowmass 2001 Working Group E2: Electron-positron Colliders from the $\phi$ to the Z, hep-ex/0201047

[16] A.E. Snyder and H.R. Quinn, Phys. Rev. D48, 2139 (1993) 